\title{
An Exploratory Study of Nonverbal Digital Video Interactive Analytic Techniques Applied to an Individual Learning Dance
}

\author{
Karen Keifer-Boyd
}

This study was an initial exploratory attempt to apply nonverbal analytic techniques to nonverbal behaviors used by an individual learning nonverbal aesthetic media (i.e., dance). The purpose of this study was to explore the possibilities, potentials, and problems of combining the Jones Visual Weighted Free Key Word Indexing System with Digital Video Interactive (DVI) technology for analyzing nonverbal behavior. These techniques employed human visual information processing and manipulation of the information as visual data in multiple configurations. The intent was to explore the usefulness of the techniques and technology as analytic tools. I applied these nonverbal analytic techniques to videorecordings of nonverbal processes used by an individual engaged in experiential learning in dance and performance arts during a one-week aesthetic education program for $\mathrm{K}-8$ teachers.

\section{Significance: Why Use Nonverbal Analytic Techniques to Study Nonverbal Learning of Nonverbal Media?}

McLeod (1991) stated that, "Doing the arts and talking about the arts are two quite different things which require very different skills" (p. 99). Beittel's (1973) seminal research focused on trying to capture the nature of the process of making art. He stressed a simultaneous view of development rather than the conventional linear view of discrete and irreversible stages of development through which each individual passes. Based on these assumptions of learning in the arts as possibly nonlinear, it is fitting to use nonverbal analytic techniques that allow both simultaneous parallel processing and sequential linear processing of information in order to study individuals involved in artmaking.

\section{Summary of Nonverbal Communication Research Design of 113 Dissertations from 1985-1991}

I reviewed nonverbal communication research designs used in dissertations from 1985 to 1991 . This provided an overview of the types of analyses that researchers have used, how they have collected data, and how they have determined categories of nonverbal behaviors. I examined the relationship between types of analyses and means to code human nonverbal behaviors that were used in nonverbal communication research in the social sciences, anthropology, dance, and in art education. The review indicated that nonverbal communication research was predominantly based on 
statistical analyses of data collected according to pre-set categories by means of tests and scales.

Research theorists have identified the need for methodology to match the purpose of the study and the complexity of the problem (Kuhn, 1962, Ittelson, et al., 1974, Hoffman, 1991). I combined the Jones System and DVI technology to investigate whether it might be more suitable for nonverbal communications research than the statistical-analytic techniques used in the past. I explored some possible ways for studying nonverbal aspects that might occur in learning a nonverbal medium such as dance.

\section{The Jones Visual Weighted Free Key Word Indexing System and Digital Video Interactive Technology}

The purpose of this study was to explore ways to visually analyze nonverbal behavior. The Jones Visual Weighted Free Key Word Indexing System, developed over the past fifteen years, assists researchers in generating grounded categories. The Jones System (1991a) draws upon the conceptual and technical bases of computer science (information retrieval, Boolean logic, weighted decisions, fuzzy logic), psychology (associativity and human visual information processing), and sociology (generation of grounded theory).

The Jones System assists the researcher in recognizing associative patterns in the data. Jones stated that, "These procedures were conceived to provide a means of conducting exploratory studies for the purposes of generating grounded categories, interpretations, and hypotheses" (1991b, p. 1). Jones explained that this process

allow[s] researchers to explore information from multiple perspectives prior to commitment to a specific focus, form of reporting, or sequence of information. . . . Coding and sorting procedures utilize visual symbolic information and overt exploratory activity designed to assist researchers' explorations of possible patterns in disparate data. (1991b, p. 15)

The Jones System also allows for the decontextualized coded data chunks to be "recontextualized in so far as the original information permits" (Jones, 1991a, p. 2).

Digital Video Interactive technology is congruent with the Jones System, since both allow for parallel processing of disparate visual data. The Jones System and DVI technology both utilize nonsequential nonhierarchical structures. Combining these two systems, I visually weighted icons and iconic sequences of digitized video built from different types of sorting procedures of a repeated confluent nonverbal behavior selected for further analysis from the data. Confluence is a term suggested by Laban to denote the harmony of movement because it is a "peculiar form of the flowing 
together of several movement constituents, which gives character to any meaningful dance-movement" (Laban, 1971, p. 31). Based on Laban's use of the term confluence, I sorted the visual icons, or codes, to find confluent movements that were repeated several times for different purposes, or in different contexts. Since the purpose of this study was to explore nonverbal analytic techniques by applying them to an example of nonverbal learning, it was important to identify movements that were similar in some way to each other and extended, or repeated over a period of time, in order to indicate possible learning. In exploratory research it is beneficial to experience the data through multiple sensory ways of knowing or perceiving so that categories are not limited by alphanumeric, linear, serial, and sequential systems of encoding and decoding the world.

Interactive digital video is a relatively new technology that is currently being explored for diverse purposes. Video and imaging technologies are continually altering the way we examine and understand reality. The ways in which video images sent from the Voyager missions were digitized and enhanced, for example, drastically altered scientists' ideas about the cosmos. This technology, however, has not been used as an analytic technique in the field of art education for exploring the process by which meanings are put together from specific parts of visual communication in and aesthetic medium. This study begins to explore how art educators could combine DVI technology with the Jones System to analyze processes and characteristics of learning and teaching nonverbal aesthetic expression.

\section{Research Design}

Research methodology influences the choice of a data collection strategy, the way the researcher perceives the data, and the appropriateness of a format or language to describe the analysis, interpretations, and findings. By choosing to explore visual analytic techniques, the researcher will see things not only as symbols, but as things in relationship to each other. This is a characteristic of human vision. Reading something visually enables one to see the whole at once and then look at the parts in relationship to the whole.

This muitimethod approach of combining the Jones Visual Weighted Free Key Word Indexing System, and various sorting procedures, with Digital Video Interactive technology was applied to the example in four distinct stages of the research process, each of which involved many steps. These four stages were: (a) planning and orientation, (b) data collection, (c) exploratory visual analysis, and (d) presentation of visual analysis and interpretations.

Figure 1. visually represents "exploratory stage three" of the example application, in this methodological study, as a spiraling process made of four loops. The word, loop, was derived from the Danish word, lob-knude, which literally meant running knot. A running knot is a helpful metaphor to describe the design. A running knot is not tied so that it stops, but rather merges things

Marilyn Zurmuehlen's Working Papers in Art Education 1993 
into a focus that forms the next loop. Interpreting patterns was the place in each loop where one loop ended and the next began. Interpreted patterns were in the form of: (a) selected videorecordings, (b) video clips as digital drawings, (c) assemblages of digital movies, and (d) interactive hypermedia. The form of interpretation influenced the form of the next transcription.

The process used for three of the four loops was based on the Jones System. It involved transcribing data into smaller pieces of information called data chunks, visually coding data, sorting visual icons, and interpreting the patterns discovered from the sorts. Each loop represented four different types of data, that is, (a) from the raw video footage, (b) from the interview with the subject of the study, (c) from the digital video enhanced by tracing around or over figures in the digitized video stills, and (d) from the movies which were assemblages of the digitized images created in loop three. Since the interview was secondary data, I transcribed the audiotape in a written form and did not use the visual techniques of the Jones System to analyze it.

Transcription began each loop since it was a way to organize data into chunks of information for further analysis. For example, in the first loop transcription was in the form of handwritten words, symbols, and simple line drawings. In the third loop, in order to transcribe, or transform, the selected repeated confluent movements from the raw video footage into digitized images I used VideoSpigot which is a combination hardware and software digital-video system for Macintosh computers. The hardware, VideoSpigot Digital-Video Frame Grabber, captures and converts the videotaped source into digital data. ScreenPlay saves the data on the hard-disk drive as a 24-bit QuickTime real-time movie. Once saved it can be edited, viewed, and placed into Adobe Premiere, a user interface for visually combining QuickTime video footage, audio recordings, animations, still images, and graphics (SuperMac Technology, 1991).

In the third loop, using a vector graphic computer drawing program called FreeHand, I visually transcribed the digitized video by drawing stick figures over th digitized video clips of the human forms, and by tracing the outlines of the main features of the human figures. There are two fundamental ways to think about drawing with a computer. One way is a geometric or object-oriented approach to drawing in which one thinks of lines connected by points. This is vector graphics. Vector refers to any line of a given magnitude and direction. The other way is bitmapped graphics which create images from a pattern of dots called pixels. Vector graphic programs recognize geometric relationships between points allowing for higher level graphic constructs called segments or groups. A group or segment is a collection of graphics that the researcher can treat as a unit. A group can be moved, scaled, rotated, or copies as a unit. It may also be joined to another group or deleted from it. Using vector graphics enabled colored codes for each of the three women. This enhanced distinctions between their forms. This enhanced distinctions between their forms. Vector graphics also enabled close analysis of the main subject's movements. For example, I separated 
one subject as a drawn form from the drawn forms of the other two women by copying, cutting, and pasting the group of lines that represented her form into a separate space on the document. (See Figure 2.) Another benefit of vector graphics compared to bitmapped images is that they require less computer memory space. The vector graphic representations conveyed more information with greater economy.

It usually took an hour to complete the drawings from each movie still, but some took considerably longer. For example, it took almost two and a half hours to draw the first series of stills from the digitized video segment of the subject performing the confluent movement. Since it was a very different position from the others that I had drawn, it took awhile to understand the pixel pattern as images of the women. In order to see the images more clearly, I watched the digitized video and paused it at the precise frame that I was drawing in enlarged dimensions. I simultaneously viewed both the live recording and the still image that I was tracing on the computer display screen.

Visual coding followed the transcription process in all but the interview loop. In the first loop, I visually coded the video transcription by creating icons with colored markers that represented the data that were placed on one side of a $5^{\prime \prime} \times 8^{\prime \prime}$ card. One set of markers was shaped so that multiple parallel lines could be drawn with one stroke. These markers enabled quickly drawn variations of a symbol. In the third loop, I visually coded the enhanced digitized video by assigning colors to groups of lines in each video still drawing. These were used as movie clips. In the fourth loop, I used colored markers to create icons that represented information in the digital movies viewed as color laser printed sets of movies on $8.5^{\prime \prime} \times 11^{\prime \prime}$ cards.

Visual sorting of the icons is part of the process of the Jones System. I did an initial sort of the visual icons generated from the transcription of the raw video footage before transcribing the interview. I sorted the visual icons, searching for patterns or themes. In the first loop, I sorted the cards of video transcription by looking for: (a) cards that had specific icons (i.e., according to different types of groupings, different interplays of verbal and nonverbal communication, levels of energy or types of facial and body emotive expression, types of movement, and various arrangements of initiating or following movements); (b) cards that had specific sets of icons (i.e., groups of three or four people in which the subject both initiated a movement and imitated another's movement); (c) frequent combinations of icons; (d) cards that had many diverse icons; and (d) cards that had combinations of icons that were repeated on several cards. This sorting process enabled me to identify repeated confluent movements. In the third loop I sorted by assembling the enhanced digitized video clips into movies, using filters and other settings to highlight patterns. (See Figure 3. for an example of sorting by adding filters to assemblages of clips.) 


\section{Summary}

Visual analysis, rather than verbal or statistical analysis, may be more suitable for studying how people learn to communicate through dance. With visual analysis the researcher can videorecord the timing, shapes, and proxemics of dance. Videorecords convey the original event more closely than do numerical or written records. It is also possible to try physically the gestures, postures, and rhythms from the visual reference of videotapes in order to feel the weight, axis, and emotion of the body. Neither movement nor visual communication needs linear and sequential systems to convey meaning.

Some of the problems addressed by anthropologists who study nonverbal communication systems by collecting photography, film, or video data, might be alleviated with the analytic and presentational possibilities of DVI technology. Hyperdocuments that include digitized video movies could: (a) reveal the process of construction and the researcher's decisions, (b) present multiple views of the nonverbal behavior studied, (c) present nonverbal analysis in nonverbal formats, and (d) provide more complete information for researchers to analyze the data further in order to build upon each other's work.

In order to study or assess the nonverbal aspects of learning development in the arts, it may be helpful to explore DVI technology. This would allow a match between the domain studied, the form that it is collected in, and the modes of thinking used to interpret or analyze the original information. This study asserts that since learning development in the arts may be studied by analyzing the process of making art, it is helpful to collect data that capture that process. This study is an initial attempt to explore the potentials of Digital Video Interactive technology combined with the Jones System for nonverbal analysis of processes involved in learning nonverbal aesthetic forms of communication.

\section{References}

Beittel, K. (1973). Alternatives for art education research: Inquiries into the making of art. Dubuque, IA: William C. Brown.

Hoffman, E. (1991). The murder quilt: A methodological study exploring selected research methods, techniques and procedures used to study material culture. Unpublished doctoral dissertation, University of Oregon, Eugene, Oregon.

Ittelson, W., Prohansky, H., Rivlin, L. \& Winkel, G. (1974). An introduction to environmental psychology. New York: Holt, Rinehart and Winston.

Jones, B. J. (1991a). Analyzing disparate data. University of Oregon. (in process). 
Jones, B. J. (1991b). Cultural implications of integrated media. Leonardo: Journal of the International Society for the Arts, Science and Technology, 24 (2), 153-158.

Kuhn, T. (1962). The structure of scientific revolutions. Chicago: University of Chicago Press.

Laban, R. (1971). Rudolf Laban speaks about movement and dance. Ullmann, L. (Ed.) Addlestone, Surrey: Laban Art of Movement Centre.

McLeod, J. (1991). Change and development. Journal of Aesthetic Eduction, 25 (2), 97-107.

SuperMac Technology. (1991). VideoSpigot si user's manual. SuperMac Technology: Sunnyvale, CA. 
The Four Loops of Stage Three: Exploratory Visual Analysis

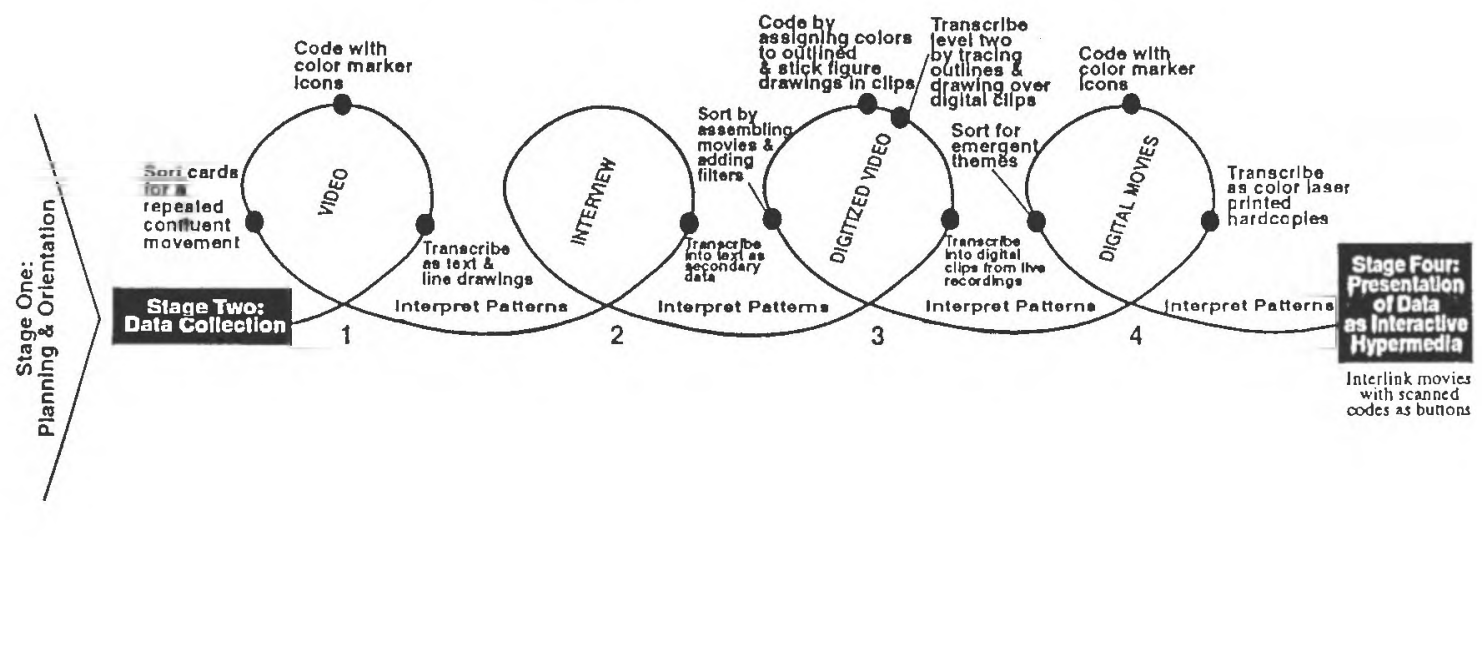

Figure 1. A diagram of the research design applied to the example in this study 

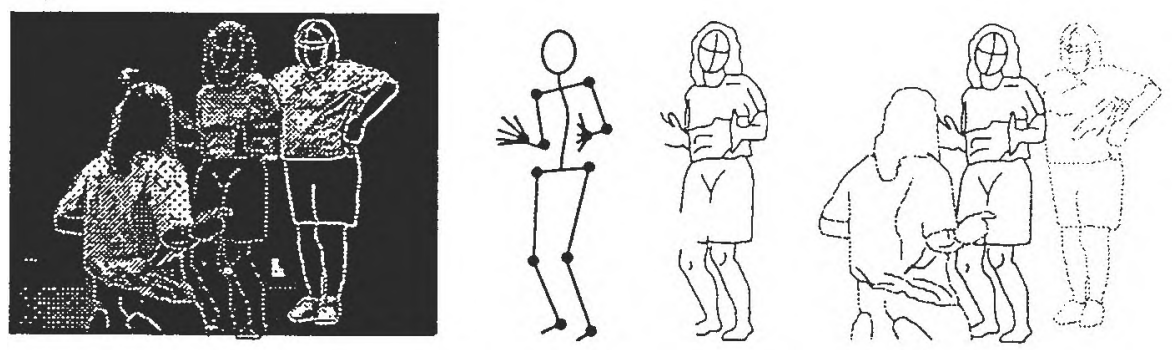

Figure 2. An example of a vector graphic transcription in the third loop of the exploratory stage of the study. 


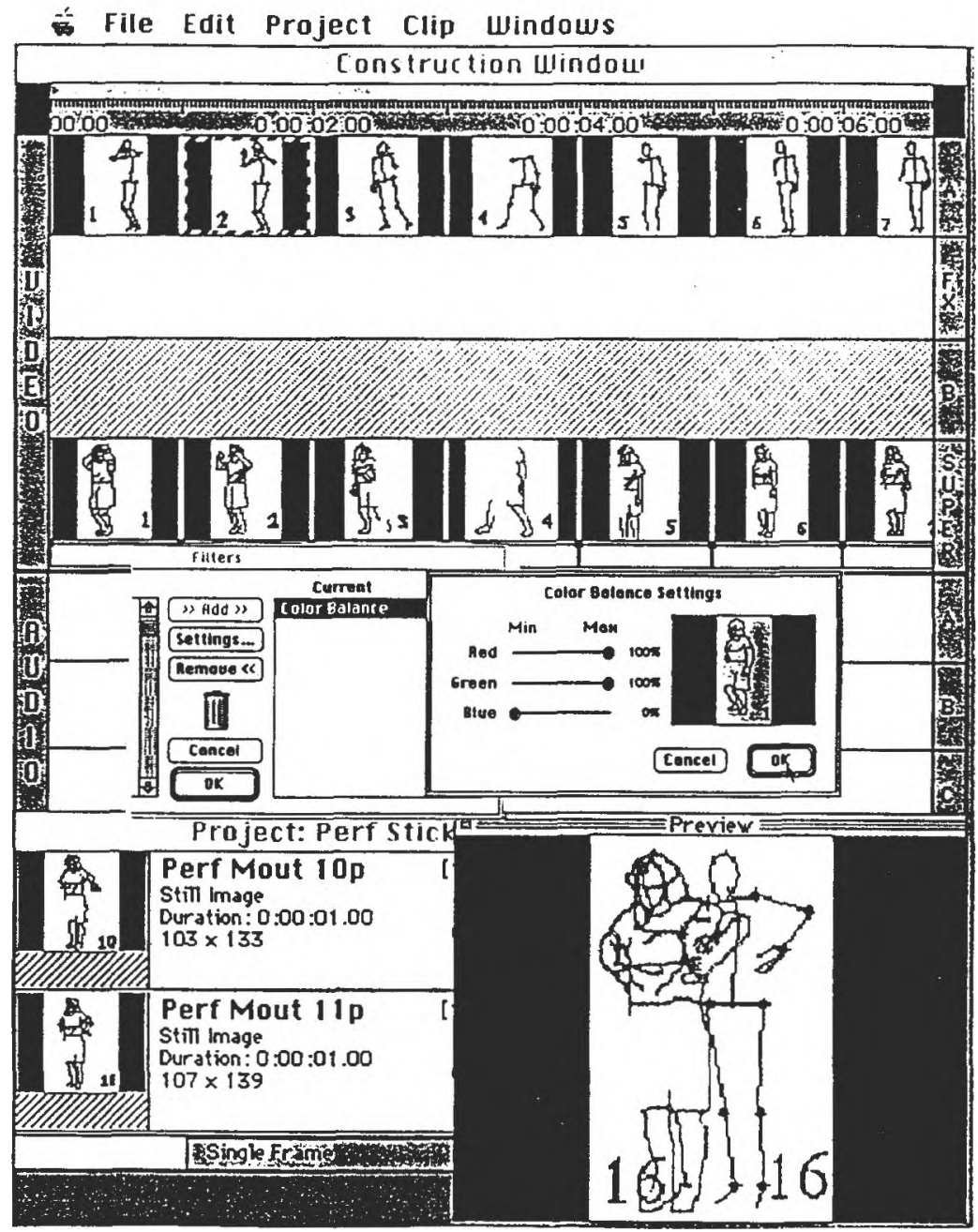

Figure 3. A Computer screen image of the movie-making software, Adobe Premier, as it was used in this study to sort data by adding filters to assembled clips. 